\title{
Histone H3/H4 tetrasome structure: analysis by spFRET microscopy
}

Anastasiia Sivkina $^{1}$, Nataliya Maluchenko ${ }^{1}$, Daria Malinina ${ }^{1}$, Aleksandra Lys ${ }^{1}$, Anna Korovina ${ }^{1}$, Alexey Feofanov $^{2}$, Vasily Studitsky ${ }^{3}$ and Mikhail Kirpichnikov ${ }^{1}$

${ }^{1}$ Faculty of Biology, Lomonosov Moscow State University, 119234 Moscow, Russia., United States, ${ }^{2}$ Biological Faculty, Lomonosov Moscow State University, Moscow, Russia, Moscow, Russia, ${ }^{3}$ Fox Chase Cancer Center, Philadelphia, PA 19111, USA., Philadelphia, Pennsylvania, United States

A nucleosome, the basic unit of eukaryotic DNA, consists of $147 \mathrm{bp}$ of DNA wrapped $~ 1.7$ times around the protein core called histone octamer, composed of a central tetramer $(\mathrm{H} 3 / \mathrm{H} 4)_{2}$ plus one $\mathrm{H} 2 \mathrm{~A} / \mathrm{H} 2 \mathrm{~B}$ dimer on either side $[1,2]$. For the implementation of fundamental biological processes, such as DNA transcription, replication, and repair, nucleosomes should dynamically dissociate and reassociate in chromatin structure. During reassociation tetramer $(\mathrm{H} 3 / \mathrm{H} 4)_{2}$ is first landed on free DNA, and only then dimers are sequentially attached to this complex. So, the tetramer serves as the structural and functional basis for nucleosomal or chromatin dynamics by initiating nucleosome assembly [3]. Thus, it is crucial to elucidate structural features of histone tetramers, which are key intermediates of many biological processes. Currently, numerous interesting data have appeared in literature but typically, these studies are done using a limited number of methods like magnetic Tweezers or computer simulation [4-6]. A promising method in this regard is spFRET (single-particle Förster Resonance Energy Transfer) spectroscopy, which has been recommended in our previous structural and dynamic studies of nucleosomes [7-9]. This work presents the use of spFRET spectroscopy approaches for the analysis of the structural features of tetramers.

Tetrasomes were assembled using 603 DNA templates and full-length recombinant Xenopus laevis histone $(\mathrm{H} 3 / \mathrm{H} 4)_{2}$ as described earlier for nucleosomes [10]. We used DNA templates with fluorescent labels placed in different positions: proximal P (+13/+91), medial $\mathrm{M}(+35 /+112)$ and distal $\mathrm{D}(+57 /+135)$ (as shown in panel 1a). As we showed earlier, selected positions provide efficient FRET in the assembled full-sized nucleosomes, and it was interesting for us to compare with FRET, which they could give only tetrasomes. spFRET spectroscopy was conducted in a free diffusion regime using the LSM710-Confocor3 system as described previously [9]. The quality of the assembled tetrasomes was analyzed by PAGE electrophoresis (panel 1b). Figure $\mathrm{c}$ shows the results of spFRET measurements of tetrasomes assembled on P, M and D (ratio template: tetramer=1:1). spFRET between labels was calculated as a proximity ratio $\left(\mathrm{E}_{\mathrm{PR}}\right)$. $\mathrm{E}_{\mathrm{PR}}$ distribution was characterized by two populations, which correspond to free DNA with a narrow high peak of low FRET (LF) and tetrasomes with an extended flat peak of high FRET (HF). The percent of particles with HF was calculated (Fig 1d). It is noteworthy that various regions of DNA wrapped differently around the tetramer. P tetrasomes had most of the particles with a high FRET. In contrast, D tetrasomes forms the smallest number of particles, and $\mathrm{M}$ tetrasomes occupy the middle position. This may indicate an asymmetry in the folding of DNA around the tetramer, which begins from one end (P part) of 603 DNA and then captures the opposite side (D). Additional experiments (panels 1e-f) with different concentrations of the tetramer showed that the introduction of an excess of the tetramer affects only the $\mathrm{M}$ and $\mathrm{D}$ parts of the tetrasome, without affecting the $\mathrm{P}$ zone. This may indicate that one part $(\mathrm{P})$ of the template DNA immediately assumes a bent conformation, which brings the fluorescent labels closer together, and an increased concentration of tetramer does not change structure. On the contrary, the remaining DNA region, apparently, remains available for the binding of the second tetramer. And when the second tetramer lands, it folds up in such a way that the labels come closer. Summarizing the experimental part, we can draw some important conclusions. 
Firstly, the use of spFRET spectroscopy using the obtained model systems is promising for studying the structural features of tetrasomes. Secondly, the asymmetric folding of nucleosomes observed in our model systems in vitro may have biological significance during the course of nuclear processes of transcription and replication, which require temporary dissociation-reassociation of the nucleosome [11].
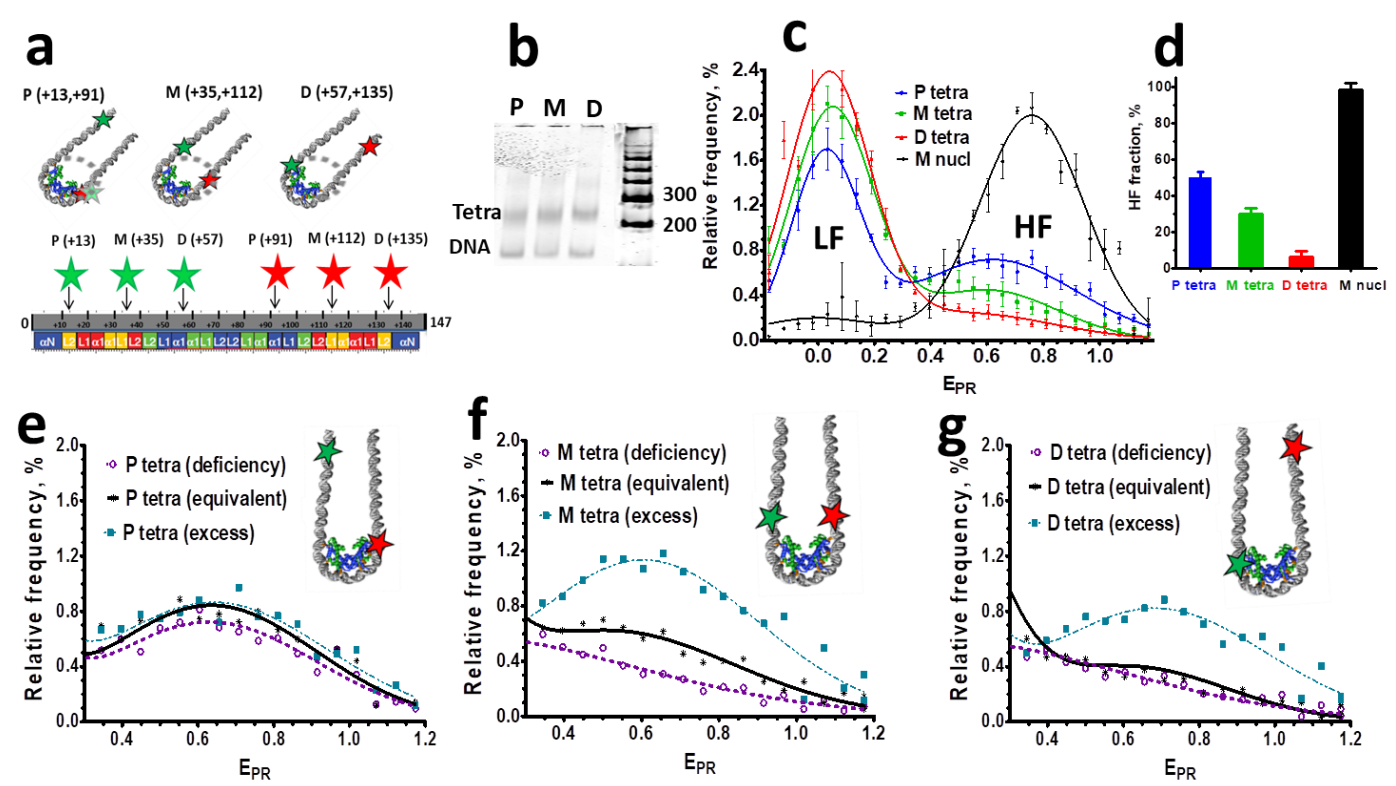

Figure 1. Structural studies of tetrasomes. A- Layout of labels in tetrasomes: proximal P $(+13 /+91)$, medial M $(+35 /+112)$ and distal D (+57/+135). Under the ruler, the location of the histone domain is schematically shown (blue - H3, green - H4, red - H2A, yellow - H2B). B- 5\% native PAGE electrophoresis of tetrasomes; Canalysis of the tetrasomes shown in panel a by spFRET (single-particle Förster Resonance Energy Transfer). spFRET between labels calculated as a proximity ratio $\left(\mathrm{E}_{\mathrm{PR}}\right)$. Typical $\mathrm{E}_{\mathrm{PR}}$-profiles are shown; high-FRET (HF) and low-FRET (LF) regions of the curves are indicated. D- quantitation of HF regions of the curves (approximated by Gaussians) shown in panel C. E-F spFRET analysis of tetrasomes with different ratio template: tetramer: 1:0,5 (deficiency), 1:1 (equivalent) and 1:2 (excess).

\section{References}

[1]. Kornberg R.D. Thomas J.O. Science. 1974. V. 184. P. 865-868.

[2]. Luger K., Mader A.W., Richmond R.K., et al. Nature. 1997. V. 389. P. 251-260.

[3]. Bina-Stein M., Simpson R.T. Cell. 1977. V. 11. N.3. P. 609-618.

[4]. Ordu O., Kremser L., Lusser A., et al. J Chem Phys. 2018. V. 148. P. 123323.

[5]. Vlijm R., Kim S.H., De Zwart P.L et al Nanoscale. 2017. V. 9. P. 1862-1870.

[6]. Onufriev A.V.,Schiessel H. Curr Opin Struct Biol. 2019. V. 56. P. 119-130.

[7]. Nilov D., Maluchenko N., Kurgina T., et al. Int J Mol Sci. 2020. V. 21.

[8]. Kantidze O.L., Luzhin A.V., Nizovtseva E.V., et al. Nat Commun. 2019. V. 10. P. 1441.

[9]. Valieva M.E., Armeev, G.A., Kudryashova, et al. Nat Struct Mol Biol. 2016. V. 23. P. 1111-1116.

[10]. Gaykalova D.A., Kulaeva, O.I., Bondarenko, V.A., et al . Methods Mol. Biol. 2009. V. 523. P. 109123.

[11]. Studies were supported by Russian Science Foundation (grant \#19-74-30003). 